\title{
Corrigendum: High yield and ultrafast sources of electrically triggered entangled-photon pairs based on strain-tunable quantum dots
}

Jiaxiang Zhang, Johannes S. Wildmann, Fei Ding, Rinaldo Trotta, Yongheng Huo, Eugenio Zallo, Daniel Huber, Armando Rastelli \& Oliver G. Schmidt

Nature Communications 6:10067 doi: 10.1038/ncomms10067 (2015); Published 1 Dec 2015; Updated 11 May 2016

This Article contains errors in the labelling of the ticks of the $x$ axis in Fig. 5. In panel a, the $x$ axis values should have been labelled ' $-10 \mathrm{~ns}$ ' and ' $10 \mathrm{~ns}$ ' rather than ' $-5 \mathrm{~ns}$ ' and ' $5 \mathrm{~ns}$ '. In panels $\mathrm{b}$ and $\mathrm{c}$, the $x$ axis values should have been labelled ' $-25 \mathrm{~ns}$ ' and ' $25 \mathrm{~ns}$ rather than ' $-10 \mathrm{~ns}$ ' and ' $10 \mathrm{~ns}$ '. The correct version of this figure appears below.
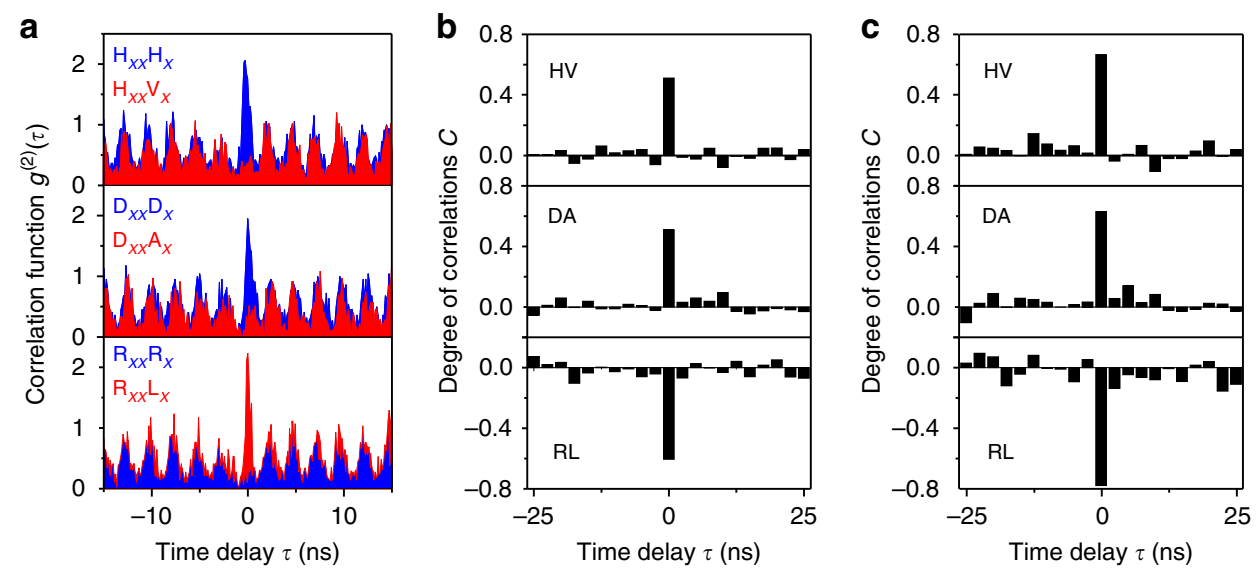

Figure 5 | Polarization correlation results from the ST-ELED under electrically pulsed injection at repetition rate of $400 \mathrm{MHz}$. (a) Normalized correlation functions for co- and cross-polarized XX and X photons in HV, DA and RL bases. (b,c) Degree of correlation $\mathrm{C}$ in given basis, in which correlation in $\mathrm{HV}$ and DA bases $(C>0)$ and anti-correlation $(C<0)$ in RL basis are obtained without temporal gate $(\Delta \tau=2.5 \mathrm{~ns})$ and with a temporal gate width $\Delta \tau=0.8 \mathrm{~ns}$ centred at 0 delay time, respectively. 